\title{
The giant pillars of the Carina Nebula
}

\author{
J. M. Rathborne ${ }^{1,2,3}$, K. J. Brooks ${ }^{1,4}$, M. G. Burton ${ }^{2}$, M. Cohen $^{5}$, and S. Bontemps ${ }^{6}$ \\ ${ }^{1}$ European Southern Observatory, Casilla 19001, Santiago 19, Chile \\ 2 School of Physics, The University of New South Wales, Sydney, NSW, 2052, Australia \\ 3 Institute for Astrophysical Research, 725 Commonwealth Avenue, Boston University, Boston, USA \\ ${ }^{4}$ Departamento de Astronomia, Universidad de Chile, Casilla 36-D, Santiago, Chile \\ 5 Radio Astronomy Laboratory, 601 Campbell Hall, University of California, Berkeley, CA 94720, USA \\ ${ }^{6}$ Observatoire de Bordeaux, BP 89, 33270 Floirac, France
}

Received 12 February 2003 / Accepted 16 October 2003

\begin{abstract}
Results are presented from a multi-wavelength study of the giant pillars within the Carina Nebula. Using near-IR data from 2MASS, mid-IR data from MSX, $843 \mathrm{MHz}$ radio continuum maps from the MOST and molecular line and continuum observations from the SEST, we investigate the nature of the pillars and search for evidence of ongoing star formation within them. Photodissociation regions (PDRs) exist across the whole nebula and trace the giant pillars, as well as many ridges, filaments and condensations $\left(A_{\mathrm{v}}>7 \mathrm{mag}\right)$. Morphological similarities between emission features at $21 \mu \mathrm{m}$ and $843 \mathrm{MHz}$ adjacent to the PDRs, suggests that the molecular material has been carved by the intense stellar winds and UV radiation from the nearby massive stars. In addition, star forming cores are found at the tips of several of the pillars. Using a stellar density distribution, several candidate embedded clusters are also found. One is clearly seen in the 2MASS images and is located within a dense core (G287.84-0.82). A search for massive young stellar objects and compact $\mathrm{H}_{\text {II }}$ regions using mid-IR colour criteria, reveal twelve candidates across the complex. Grey-body fits to SEDs for four of these objects are suggestive of OB-stars. We find that massive star formation in the Carina Nebula is occurring across the whole complex and confirm it has been continuous over the past 3 Myrs.
\end{abstract}

Key words. ISM: structure - ISM: lines and bands - ISM: molecules - $\mathrm{H}_{\text {II }}$ regions - dust, extinction - stars: formation

\section{Introduction}

In many cases, young stars have been found at the tips of giant pillars that point toward a more evolved massive star cluster (e.g. the elephant trunks of the Eagle Nebula; McCaughrean $\&$ Andersen 2002). The formation of such pillars can readily occur if a dense core within a giant molecular cloud (GMC) is exposed to the intense stellar winds and radiation fields from a nearby massive star cluster. The core would shield the column of molecular material behind it, in a direction pointing away from the cluster. Subsequently, the more exposed parts of the GMC would be swept up around this column or be completely irradiated away.

It is not clear if such a drastic change in the structure of a GMC can affect its star formation capacity. There is growing evidence to suggest that the tips of pillars are prime sites for ongoing star formation (Jiang et al. 2002; Stanke et al. 2002; McCaughrean \& Andersen 2002). However, there is much debate over whether this type of star formation has been triggered by external processes, or whether it has spontaneously formed. It is uncertain if we can even distinguish between the two.

Send offprint requests to: J. M. Rathborne, e-mail: jmr@phys.unsw.edu.au
Several giant pillars pointing toward the massive clusters within the Carina Nebula have recently been discovered at midinfrared (mid-IR) wavelengths, the largest extending $\sim 25 \mathrm{pc}$ (Smith et al. 2000). Bright IR emission condensations are located at the tips of several of these pillars, and may correspond to sites where star formation has been triggered by the interactions with the surrounding young massive star clusters (Smith et al. 2000).

Many large molecular clouds are associated with the Carina Nebula (Zhang et al. 2001; Brooks et al. 1998; Whiteoak \& Otrupcek 1984; de Graauw et al. 1981). These clouds lie close to several massive star clusters, in particular Bochum 10 and 11, Collinder 228 and Trumpler 14, 15 and 16 (hereafter the cluster abbreviations Bo, Co and $\mathrm{Tr}$ will be used). These clusters contain a combined total of 64 O-type stars including one of the most massive and spectacular stars known, $\eta$ Car (Feinstein 1995). Located at a distance of $2.2 \mathrm{kpc}$ (Tovmassian 1995), the Carina Nebula is an excellent region in which to study the effect massive stars have on their natal GMC.

The giant pillars are located in the relatively unstudied southern region of this nebula, at a greater distance from the most influential clusters. The stellar winds and radiation fields may be less destructive here, making the pillars prospective 
sites for ongoing star formation. It is the aim of this paper to investigate the nature of the interstellar medium within, and surrounding these pillars, and in particular to determine if there exists any evidence for ongoing star formation within them.

\section{The data}

\subsection{MSX on-line database}

Images between $8-21 \mu \mathrm{m}$ were obtained from the $M S X$ database $^{1}$, and cover four discrete bands; Band A (6.8$10.8 \mu \mathrm{m})$, Band $C(11.1-13.2 \mu \mathrm{m})$, Band $D(13.5-15.9 \mu \mathrm{m})$ and Band $E(18.2-25.1 \mu \mathrm{m})$. These bands will be hereafter referred to as the $8,12,14$, and $21 \mu \mathrm{m}$ bands respectively. The angular resolution of the images $(F W H M)$ is $20^{\prime \prime}$ with an astrometric accuracy of $\sim 2^{\prime \prime}$.

Over the four MSX bands, the emission arises from either fluorescently excited polycyclic aromatic hydrocarbon (PAH) molecules or black-body emission from heated dust grains (150-400 K). Emission from the family of PAH features (6.2, $7.7,8.7,11.3,12.7$ and $16.4 \mu \mathrm{m})$, as well as several emission plateaus caused by small PAH molecules $(6-9 \mu \mathrm{m}, 11-13 \mu \mathrm{m}$ and $15-20 \mu \mathrm{m})$ are all contained within the 8, 12 and $14 \mu \mathrm{m}$ bands (Verstraete et al. 2001). The emission seen in the $21 \mu \mathrm{m}$ band generally arises from heated dust, and is useful in revealing the locations of heavily obscured young stars.

The MSX Point Source Catalogue (PSCv1.0) provided position and flux measurements for point sources within the Carina Nebula (over the extent shown in Fig. 1). A total of 526 point sources were found, however, the majority are only detected in the higher sensitivity $8 \mu \mathrm{m}$ band. For the discussion presented in Sect. 4.2, a subset of this larger catalogue was used. Sources were extracted from the PSC if they had reliable fluxes in each of the 8-, 14- and $21 \mu \mathrm{m}$ bands (corresponding to quality flags 3 or 4 ). This reduced the list to a total of 33 sources.

\subsection{MOST on-line database}

The radio continuum data presented here were obtained as part of the Molonglo Galactic Plane Survey (MGPS) made with the Molonglo Observatory Synthesis Telescope (MOST) ${ }^{2}$. The centre frequency of the MOST is $843 \mathrm{MHz}$, with a bandwidth of $3 \mathrm{MHz}$. The synthesized beam is $43^{\prime \prime} \times 43^{\prime \prime} \operatorname{cosec}|\delta|$. For more details of the specifications of the MOST see Mills (1981) and Robertson (1991).

\subsection{SEST observations}

\subsubsection{Spectral line observations}

Spectral line observations were obtained toward four mid-IR condensations (G287.73-0.92, G287.84-0.82,

\footnotetext{
${ }^{1}$ For a full description of the MSX satellite, the astrophysical experiments and the observing techniques see Mill et al. (1994); Price et al. (1996); Egan et al. (1998); Price et al. (2001).

${ }^{2}$ http://www.physics.usyd.edu.au/astrop/most/
}

G287.93-0.99 and G288.07-0.80; Smith et al. 2000) using the 15-m Swedish-ESO Submillimetre Telescope (SEST) ${ }^{3}$ at La Silla Observatory, Chile. The condensations were observed in six molecular lines: ${ }^{12} \mathrm{CO}(1-0),{ }^{12} \mathrm{CO}(2-1)$, ${ }^{13} \mathrm{CO}(2-1), \mathrm{C}^{18} \mathrm{O}(1-0), \mathrm{CS}(3-2)$ and $\mathrm{CS}(2-1)$. Details of the observations are given in Table 1.

Both the IRAM-built $230 / 115 \mathrm{GHz}$ and the SESIS $150 / 100 \mathrm{GHz}$ receivers were used. Transitions were observed simultaneously where possible in single side band mode (SSB), with the receivers connected to a narrow-band $(43 \mathrm{MHz})$ Acousto-Optical Spectrometer (AOS).

Position-switching mode was used for all observations, with $\eta$ Car used as the signal-free reference position. An offsource position was taken after every second on-source integration. Chopper wheel calibration was performed every $10 \mathrm{~min}$, to obtain atmosphere-corrected antenna temperatures. The telescope pointing and sub-reflector focusing were checked regularly using suitably bright nearby $\mathrm{SiO}$ masers. We estimate the pointing accuracy to be better than $10^{\prime \prime}$ and adopt the standard SEST value of 10 per cent uncertainty in the temperature scale.

Observations toward the condensations were obtained using a $20^{\prime \prime}$ pointing grid and intended to cover the peak of the emission. However, in several cases data were only obtained over the tip of much larger structures.

All spectra were processed using GILDAS software (Buisson et al. 1999). Baselines were initially removed from each spectrum and the temperature scale converted to mainbeam brightness temperature using the values for the main beam efficiencies $\left(\eta_{\mathrm{mb}}\right)$ given in Table 1 . For comparison between data sets with differing beam sizes, the data were smoothed to the larger beam-size on a similarly sized grid. The beam size of the SEST at each frequency, the average system temperatures during the observations, and the average rms noise per spectral channel are also given in Table 1.

\subsubsection{Continuum observations}

Observations of 1.2-mm continuum emission were also obtained with the SEST using the 37 channel Imaging Bolometer Array (SIMBA). The FWHM of each element is $23^{\prime \prime}$ within the array, with a separation between each of $44^{\prime \prime}$. The observations were conducted in July 2002 in the fast-scanning mode $\left(80 \operatorname{arcsec~s}^{-1}\right)$. Data for a single point source in the Carina Nebula (G287.87-1.36) were obtained using a map size of $480^{\prime \prime} \times 240^{\prime \prime}$ (az $\times$ elev). To complete this map, 31 subscans were required with each sub-scan separated by $8^{\prime \prime}$. Each map took $\sim 4$ min with the map repeated 6 times to improve the signal to noise. The pointing and sub-reflector focusing were checked prior to the observations with sky-dip calibrations performed both before and after the mapping. Observations of Uranus were also obtained for flux calibration. The average opacity during the observations was $\sim 0.14$.

All maps were reduced by applying the opacity corrections, fitting and subtracting a baseline, and removing the correlated

\footnotetext{
3 The SEST is operated jointly by ESO and the Swedish National Facility for Radio Astronomy, Onsala Space Observatory, Chalmers University of Technology.
} 


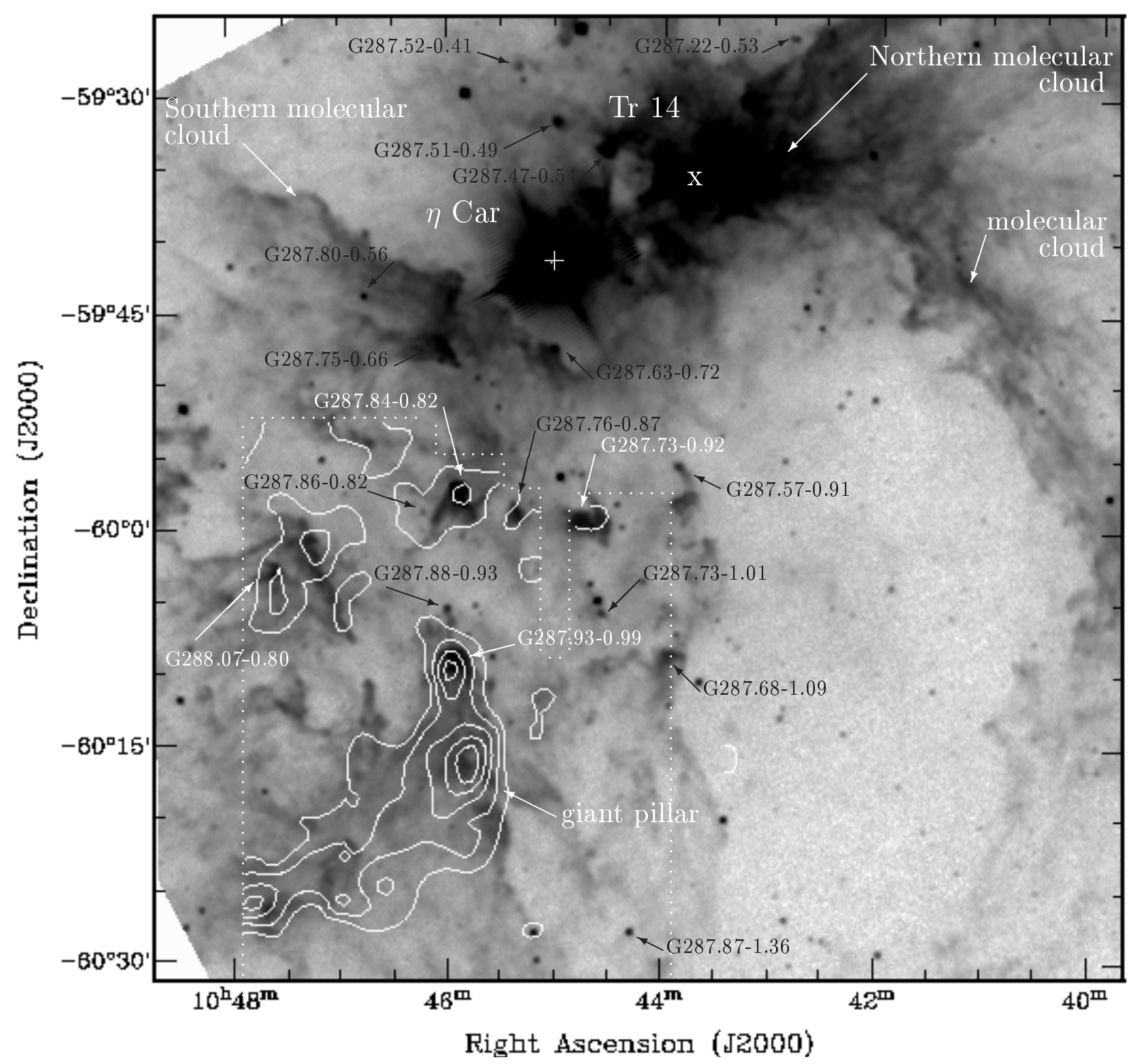

Fig. 1. The Carina Nebula in $8 \mu \mathrm{m}$ emission (logarithmic grey-scale) with contours of visual extinction (levels are 7.5, 9.5, 11.5, 13.5 and $15.5 \mathrm{mag})$. Marked on the figure are the locations of $\eta \operatorname{Car}(+), \operatorname{Tr} 14(\mathrm{x})$, the southern and northern molecular clouds, and the additional giant pillar and molecular cloud identified by Zhang et al. (2001). The dotted region outlines the extent of the extinction map. The four condensations labelled (in white) G287.73-0.92, G287.84-0.82, G287.93-0.99 and G288.07-0.80 are discussed in Sect. 3.5. The point sources labelled in black mark the sources discussed in Sect. 4.

sky noise. Maps were flux calibrated using the conversion factor obtained from the Uranus observations. The rms noise level in the final map was $9 \mathrm{mJy}$. All data reduction was achieved within the MOPSI package ${ }^{4}$.

\subsection{MASS on-line database}

Near-IR images were obtained from the Two Micron All Sky Survey (2MASS) ${ }^{5}$ Second Incremental Data Release. The images cover the three near-IR bands: $J(1.24 \mu \mathrm{m}), H(1.66 \mu \mathrm{m})$,

\footnotetext{
${ }^{4}$ MOPSI was developed by R. Zylka (Astrophysikalisches Institut, Universität zu Köln, Germany).

${ }^{5}$ see http://www.ipac.caltech.edu/2mass/index.html
}

and $K_{\mathrm{S}}(2.16 \mu \mathrm{m})$ down to limiting magnitudes of $15.8,15.1$ and 14.3 respectively. The resolution of the final images is $2^{\prime \prime}$.

\section{The nature of the pillars}

This section aims to identify, and determine the nature of, the pillars within the southern Carina Nebula. We will attempt to trace the extent of the gas and dust within the region, determine how the radiation from the nearby clusters is penetrating through the GMC, locate ionization fronts, and attempt to characterise the properties of regions where star formation may be occurring. 
Table 1. Observing parameters for the molecular line data obtained with the SEST. $t_{\text {int }}$ refers to the integration time, $H P B W$ is the half-power beam-width, $\eta_{\mathrm{mb}}$ is the main beam efficiency, $T_{\mathrm{sys}}$ is the average system temperature, and $\sigma$ is the average rms noise per spectral channel for the observations.

\begin{tabular}{cccccccc}
\hline \hline Transition & $\begin{array}{c}\text { Frequency } \\
\mathrm{GHz}\end{array}$ & Date & $\begin{array}{c}t_{\text {int }} \\
\mathrm{s}\end{array}$ & $\begin{array}{c}H P B W \\
\operatorname{arcsec}\end{array}$ & $\begin{array}{c}\eta_{\mathrm{mb}} \\
T_{\text {sys }}\end{array}$ & $\begin{array}{c}\sigma \\
\mathrm{K}\end{array}$ \\
\hline${ }^{12} \mathrm{CO}(1-0)$ & 115.217 & Oct. 2000 & 30 & 45 & 0.70 & 529 & 0.7 \\
${ }^{12} \mathrm{CO}(2-1)$ & 230.537 & Oct. 2000 & 30 & 23 & 0.50 & 625 & 0.7 \\
$\mathrm{C}^{18} \mathrm{O}(1-0)$ & 109.782 & Mar. 2002 & 120 & 49 & 0.72 & 321 & 0.4 \\
${ }^{13} \mathrm{CO}(2-1)$ & 220.398 & Mar. 2002 & 120 & 24 & 0.52 & 488 & 0.5 \\
$\mathrm{CS}(2-1)$ & 97.981 & Jan. 2002 & 60 & 52 & 0.73 & 337 & 0.4 \\
$\mathrm{CS}(3-2)$ & 146.969 & Jan. 2002 & 60 & 34 & 0.66 & 433 & 0.5 \\
\hline
\end{tabular}

${ }^{a}$ The observations at these frequencies for source G287.93-0.99 were obtained in Oct. 2000.

\subsection{Photodissociation regions}

Figure 1 shows the Carina Nebula in $8 \mu \mathrm{m}$ emission. This image reveals many regions of extended $8 \mu \mathrm{m}$ emission in addition to several bright isolated features. This band is dominated by PAH emission and is tracing photodissociation regions (PDRs). These occur within a visual extinction $\left(A_{\mathrm{V}}\right) \sim$ $1 \mathrm{mag}$ on the surface of molecular material, where the UV radiation has penetrated and is heating the material (Hollenbach \& Tielens 1999).

Evident to the northwest of this nebula is a prominent $8 \mu \mathrm{m}$ feature which corresponds to a component of the GMC known as the northern molecular cloud. The bright emission south of $\eta$ Car traces the edge of the southern molecular cloud. Located further to the south is the giant pillar identified by Smith et al. (2000). These clouds are all identified in the large scale $\mathrm{CO}(4-3)$ and $[\mathrm{CI}]$ survey of Zhang et al. (2001). These transitions are tracers of heated gas $(\sim 50 \mathrm{~K})$, dense gas ( $n \sim 10^{5} \mathrm{~cm}^{-3}$ ), and PDRs, respectively, and confirm that the $8 \mu \mathrm{m}$ emission is tracing the outer PDR layers of the molecular clouds.

Figure 1 also illustrates the mid-IR sources identified by Smith et al. (2000; G287.73-0.92, G287.84-0.82, G287.93-0.99 and G288.07-0.80). Many other point sources are also found across the complex, and are located at the tips of more extended $8 \mu \mathrm{m}$ emission (e.g. G287.57-0.91 and G287.88-0.93). Their morphology is consistent with a dense core being irradiated by $\operatorname{Tr} 14$ and $\operatorname{Tr} 16$.

\subsection{Visual extinction}

Extinction maps were generated for the southern Carina Nebula using stars within the 2MASS PSC. Estimates of the visual extinction were obtained for each star detected in the $J H K_{\mathrm{s}}$-bands, by dereddening each to an average main sequence colour in the $(J-H)$ vs. $\left(H-K_{\mathrm{s}}\right)$ plane. The visual extinctions obtained were then used to construct a global extinction map for the region (contours in Fig. 1, the extent of the map is marked with the dotted line).

Clearly seen as a region of high visual extinction is the giant pillar, where the morphology of the extinction matches the $8 \mu \mathrm{m}$ emission extremely well. In addition to this giant pillar, other regions of high visual extinction are also found to correspond to $8 \mu \mathrm{m}$ features.

The visual extinction across the giant pillar ranges from 7-15 mag and can be used to estimate the $\mathrm{H}_{2}$ column density within it. Applying the relation, $N\left({ }^{13} \mathrm{CO}\right) \simeq 2.7 \times$ $10^{15} A_{\mathrm{v}}\left(\mathrm{cm}^{-2}\right.$; Frerking et al. 1982) and converting to $N\left(\mathrm{H}_{2}\right)$ using the constant of proportionality of $4 \times 10^{5}$ (Lada et al. 1994), the $\mathrm{H}_{2}$ column density was found to range between $9-19 \times 10^{21} \mathrm{~cm}^{-2}$.

\subsection{Heated dust}

Figure 2 shows the $21 \mu \mathrm{m}$ and $843 \mathrm{MHz}$ emission across the Carina Nebula. Of note, is the striking morphological similarities of these emission features across the complex.

Strong $21 \mu \mathrm{m}$ emission surrounds $\eta$ Car and Tr 14 with diffuse emission found in the south. Several bright point sources are also evident, in particular the bright source associated with the $8 \mu \mathrm{m}$ condensation G287.84-0.82. As mentioned previously, extended emission seen within the $21 \mu \mathrm{m}$ band generally arises from heated dust, while $21 \mu \mathrm{m}$ point sources identify deeply embedded stars.

By matching the expected surface brightness of an optically thick black-body with the observed flux from regions showing strong $21 \mu \mathrm{m}$ emission, we find a dust temperature of $42-$ $44 \mathrm{~K}$. This temperature is in good agreement with other estimates $(40 \mathrm{~K})$ obtained from IRAS data in the $\operatorname{Tr} 14$ region (Harvey et al. 1979).

\subsection{Ionization fronts}

Radio continuum emission is widespread throughout the Carina Nebula, with the brightest concentrations known as Car I and Car II (Brooks et al. 2001; Whiteoak 1994; Retallack 1983; Gardner \& Morimoto 1968).

Extending from Car I is a long filament of weaker emission. Similar features are also seen in the southern part of the nebula, and are labelled in Fig. 2. All of these features are coincident with $21 \mu \mathrm{m}$ emission, and in the majority of cases, are found adjacent to regions of bright $8 \mu \mathrm{m}$ emission and peaks in the visual extinction map. This morphology is consistent with the ionization fronts heating the dust and creating the widespread PDRs. 


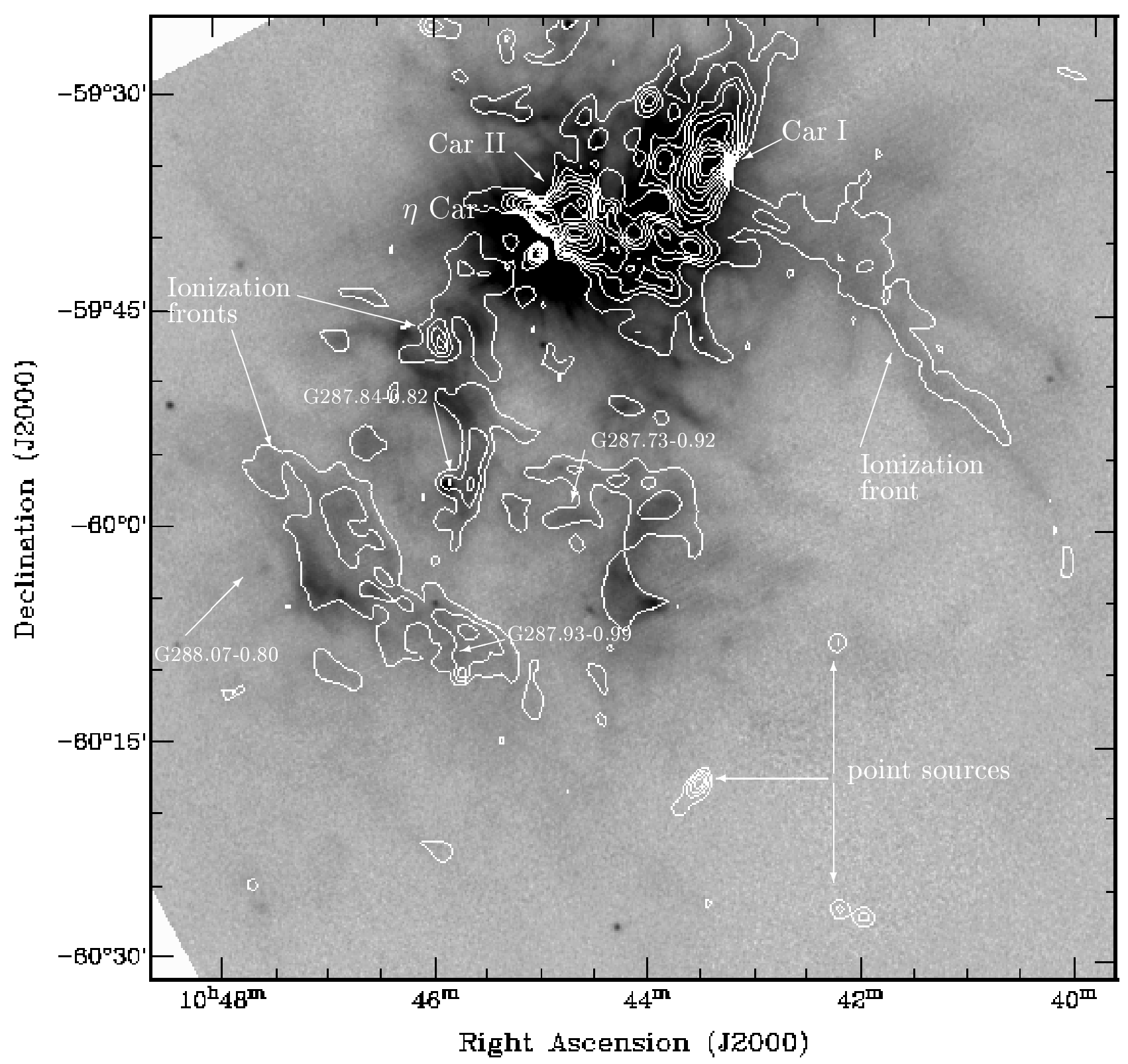

Fig. 2. The Carina Nebula in $21 \mu \mathrm{m}$ emission (logarithmic grey-scale) with contours of $843 \mathrm{MHz}$ radio continuum emission (levels are 0.04 , $0.14,0.24,0.34,0.5,0.7,0.9,1.1,1.3,1.5 \mathrm{Jy} / \mathrm{beam})$. Marked in this figure is the location of $\eta$ Car as well as several ionization fronts (including Car I and Car II), and the four condensations; G287.73-0.92, G287.84-0.82, G287.93-0.99 and G288.07-0.80.

Compact sources are also evident across the complex. The majority of these have no mid-IR counterparts (marked as "point sources" in Fig. 2), suggesting they correspond to unresolved background galaxies (Cohen \& Green 2001). The exception is G287.84-0.82, which has an extremely strong mid-IR counterpart. This object is likely to be a compact $\mathrm{H}$ II region.

\subsection{Properties of the four bright mid-IR condensations}

Molecular material was detected toward each of the four mid-IR condensations, G287.73-0.92, G287.84-0.82, G287.93-0.99 and G288.07-0.80. Their integrated ${ }^{12} \mathrm{CO}(2-1)$ intensity maps, show the morphology of the molecular structures match well with the corresponding $8 \mu \mathrm{m}$ emission (Fig. 3), further confirming the presence of PDRs on their surfaces. For G288.07-0.80 however, the geometry of the molecular material differs considerably from the $8 \mu \mathrm{m}$ emission. While the morphology of the molecular gas is consistent with irradiation from the direction of the clusters $\operatorname{Tr} 14$ and $\operatorname{Tr} 16$, the PDR appears to point in a different direction. Interestingly, this corresponds to the direction of the Bo 11 cluster.

Figure 4 shows the line profiles for each condensation, in each of the molecular transitions observed. The peak temperature $(T)$, central velocity $(V)$ and line-width $(\Delta V)$ for these profiles were determined from Gaussian fits, the results of which are given in Table 2.

The molecular condensations show a range in velocities of $\sim 15 \mathrm{~km} \mathrm{~s}^{-1}$. This is consistent with the velocity dispersion of molecular clumps, and motion of the ionized gas, observed 

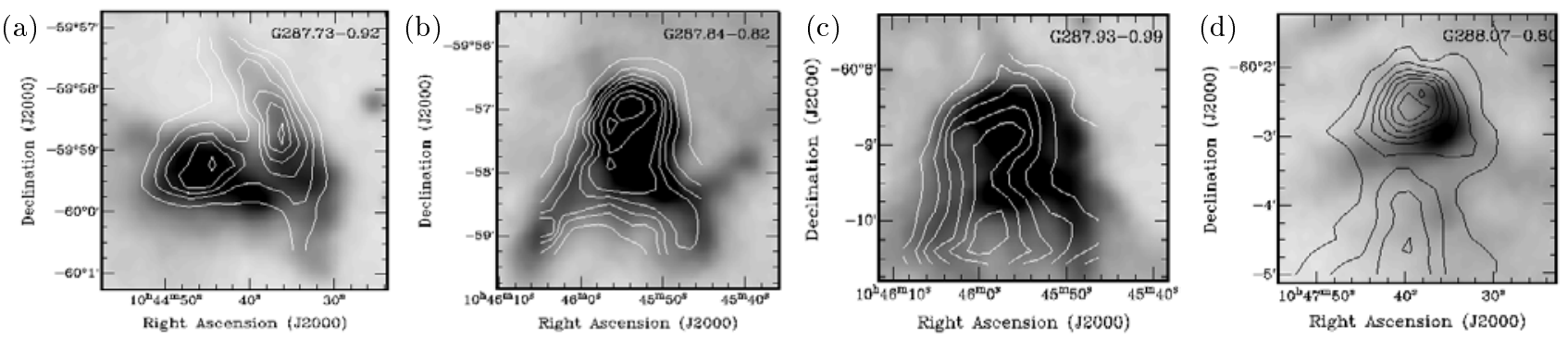

Fig. 3. $8 \mu \mathrm{m}$ emission in grey-scale with contours of integrated ${ }^{12} \mathrm{CO}(2-1)$ emission for the condensations. a) G287.73-0.92: Integrated from -25 to $-32 \mathrm{~km} \mathrm{~s}^{-1}$, levels are $20,35,50,65,80,95 \mathrm{~K} \mathrm{~km} \mathrm{~s}^{-1}$; b) G287.84-0.82: Integrated from -12 to $-16 \mathrm{~km} \mathrm{~s}^{-1}$, levels are 20 , 40, 60, 80, 100, 140, $180 \mathrm{~K} \mathrm{~km} \mathrm{~s}^{-1}$; c) G287.93-0.99: Integrated from -18 to $-22 \mathrm{~km} \mathrm{~s}^{-1}$, levels are $30,50,70,90,110,130 \mathrm{~K} \mathrm{~km} \mathrm{~s}{ }^{-1}$; d) G288.07-0.80: Integrated from -19 to $-24 \mathrm{~km} \mathrm{~s}^{-1}$, levels are $15,30,45,60,75,90,105 \mathrm{~K} \mathrm{~km} \mathrm{~s}^{-1}$.

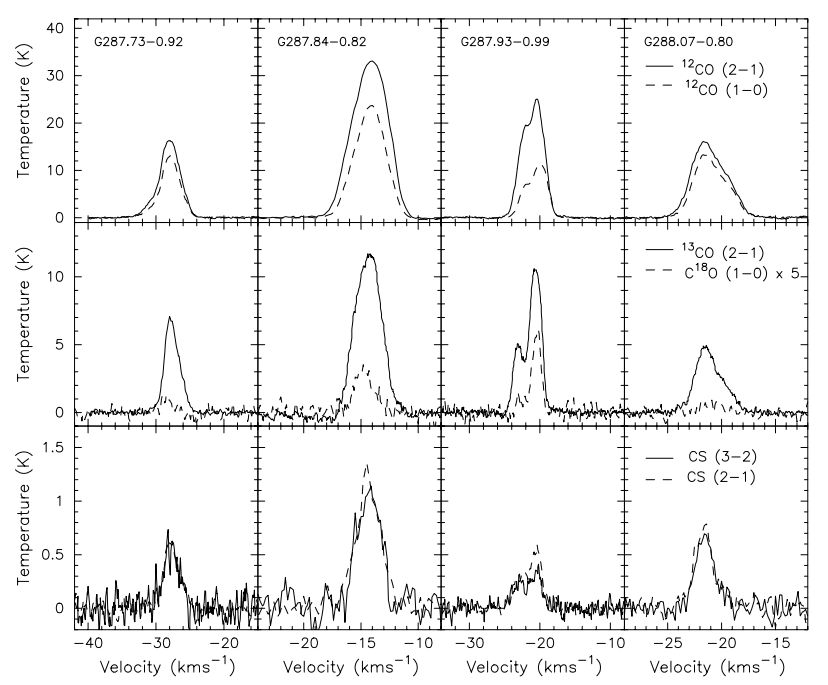

Fig. 4. Line profiles spatially integrated over each condensation. Left to right: G287.73-0.92, G287.84-0.82, G287.93-0.99 and G288.07-0.80. Top to bottom: ${ }^{12} \mathrm{CO}(2-1)$ and ${ }^{12} \mathrm{CO}(1-0) ;{ }^{13} \mathrm{CO}(2-1)$ and $\mathrm{C}^{18} \mathrm{O}(1-0)$; $\mathrm{CS}(3-2)$ and $\mathrm{CS}(2-1)$ emission. The $\mathrm{C}^{18} \mathrm{O}(1-0)$ profiles are multiplied by 5 to make them visible. Note the different temperature scales and velocity ranges.

within the Keyhole Nebula ( $20 \mathrm{~km} \mathrm{~s}^{-1}$; Cox \& Bronfman 1995). This suggests there is also a strong interaction between the winds and radiation from the stellar clusters and these molecular condensations.

Estimates of the physical properties of the condensations were determined from the profiles under the assumption of local thermal equilibrium (LTE). Values for the excitation temperatures, line ratios, opacities, masses, and average hydrogen densities for each condensations are listed in Table 3. The ratio of the integrated intensity of the ${ }^{12} \mathrm{CO}(2-1)$ and (1-0) emission ranged between 1-2, and is consistent with optically thick ${ }^{12} \mathrm{CO}$ emission. In addition, excitation temperatures were found to range between $20-40 \mathrm{~K}$.

Ratios of the ${ }^{12} \mathrm{CO}(2-1)$ and ${ }^{13} \mathrm{CO}(2-1)$ emission ranged between $2-5$, suggesting that the ${ }^{13} \mathrm{CO}(2-1)$ transition is optically thin. To estimate the opacity and column density of this material, a constant excitation temperature of $35 \mathrm{~K}$ was used. Opacities ranged from 0.16-0.4, confirming the emission is optically thin. $\mathrm{H}_{2}$ column densities were determined to be $7-22 \times 10^{21} \mathrm{~cm}^{-2}$, and are consistent with values determined when using the estimated $A_{\mathrm{v}}$ derived from the star colours $\left(9-19 \times 10^{21} \mathrm{~cm}^{-2}\right)$.

Estimates of the masses were calculated using the column densities determined from the ${ }^{13} \mathrm{CO}$ emission (LTE mass). This calculation assumes the integrated intensity of the emission is proportional to the $\mathrm{H}_{2}$ column density with a constant of proportionality of $4 \times 10^{5}$ (Lada et al. 1994). Masses ranged from 10-45 $M_{\odot}$ for the condensations, however, these may be under-estimated by as much as a factor of 3 . The Virial mass was estimated using the line-width of the ${ }^{13} \mathrm{CO}$ line, which is assumed to be a measure of the overall motion of the gas and hence the mass. Values of the Virial mass for the condensations ranged between 80-200 $M_{\odot}$. These are significantly greater than the LTE masses (even considering the latter may be underestimating the true masses), implying that the condensations are probably not gravitationally bound but instead confined by external pressure.

The average $\mathrm{H}_{2}$ density was determined assuming the size along the line-of-sight was the average of the extent in right ascension and declination. Estimates ranged between 4-17 $\times$ $10^{3} \mathrm{~cm}^{-3}$ and represent lower limits to the actual values. In addition, the presence of CS emission suggests the material has gas densities in excess of $10^{4} \mathrm{~cm}^{-3}$. The properties described here are consistent with molecular cores found in other regions of massive star formation (Zinchenko et al. 1998).

\section{Star formation within the pillars}

This section investigates the star formation activity across the Carina Nebula, by indentifying candidate embedded clusters and young stellar objects (YSOs).

\subsection{Young clusters}

All stars within the 2MASS PSC were dereddened. As previously discussed, those detected in the $J H$ and $K_{\mathrm{s}}$-bands were dereddened using an average main sequence colour. For those stars only detected in the $H$ - and/or $K_{\mathrm{s}}$-bands, the extinction was estimated using nearby stars in the global extinction map. From these dereddened $K_{\mathrm{s}}$-band magnitudes $\left(K_{\text {corr }}\right)$ a stellar number density map was created for stars with $K_{\text {corr }}>12$ mag. Choosing this cut-off corresponds to the completeness limit from the 2MASS photometry for an $A_{\mathrm{v}}$ of $25 \mathrm{mag}$. 
Table 2. Properties of the spatially integrated profiles seen toward the condensations. These were obtained from Gaussian fits to the profiles shown in Fig. 4. The parameters include; the central velocity $\left(V, \mathrm{~km} \mathrm{~s}^{-1}\right)$, peak temperature $(T, \mathrm{~K})$ and line-width $\left(\Delta V, \mathrm{~km} \mathrm{~s}^{-1}\right)$.

\begin{tabular}{|c|c|c|c|c|c|c|c|c|c|c|c|c|c|c|c|c|c|}
\hline \multicolumn{3}{|c|}{${ }^{12} \mathrm{CO}(1-0)$} & \multicolumn{3}{|c|}{${ }^{12} \mathrm{CO}(2-1)$} & \multicolumn{3}{|c|}{$\mathrm{C}^{18} \mathrm{O}(1-0)$} & \multicolumn{3}{|c|}{${ }^{13} \mathrm{CO}(2-1)$} & \multicolumn{3}{|c|}{$\mathrm{CS}(2-1)$} & \multicolumn{3}{|c|}{$\mathrm{CS}(3-2)$} \\
\hline V & $T$ & $\Delta V$ & V & $T$ & $\Delta V$ & $V$ & $T$ & $\Delta V$ & V & $T$ & $\Delta V$ & V & $T$ & $\Delta V$ & $V$ & $T$ & $\Delta V$ \\
\hline \multicolumn{18}{|c|}{ G287.73-0.92 } \\
\hline-31.5 & 1.0 & 2.1 & -31.5 & 1.7 & 1.8 & & & & -30.3 & 0.4 & 1.5 & & & & & & \\
\hline \multirow[t]{2}{*}{-27.8} & 12.8 & 3.2 & -27.9 & 16.4 & 3.4 & -28.4 & 0.2 & 2.2 & -28.1 & 6.0 & 1.7 & -27.9 & 0.6 & 2.3 & -27.9 & 0.6 & 2.5 \\
\hline & & & & & & & & & -26.7 & 3.2 & 2.1 & -25.8 & 0.2 & 1.2 & -26.0 & 0.2 & 0.8 \\
\hline \multicolumn{18}{|c|}{ G287.84-0.82 } \\
\hline-14.2 & 23.8 & 3.2 & -14.2 & 34.1 & 3.6 & -14.7 & 0.6 & 1.9 & -14.4 & 12.0 & 2.7 & -14.4 & 1.2 & 2.5 & -14.3 & 1.1 & 2.4 \\
\hline \multicolumn{18}{|c|}{ G287.93-0.99 } \\
\hline-22.1 & 6.2 & 2.0 & -22.4 & 15.3 & 2.1 & -22.6 & 0.2 & 1.6 & -23.1 & 5.0 & 1.6 & -22.7 & 0.3 & 2.2 & -22.9 & 0.2 & 2.4 \\
\hline-19.8 & 11.5 & 2.3 & -20.3 & 23.6 & 2.4 & -20.4 & 1.2 & 1.4 & -20.6 & 11.0 & 2.0 & -20.4 & 0.5 & 1.8 & -20.4 & 0.3 & 1.7 \\
\hline \multicolumn{18}{|c|}{ G288.07-0.80 } \\
\hline-21.6 & 13.0 & 2.6 & -21.7 & 15.5 & 2.7 & -20.9 & 0.1 & 2.2 & -21.5 & 4.7 & 2.2 & -21.7 & 0.8 & 1.9 & -21.6 & 0.7 & 2.1 \\
\hline-19.3 & 5.4 & 2.2 & -19.4 & 6.6 & 2.3 & & & & -19.5 & 1.5 & 1.9 & -19.6 & 0.1 & 1.2 & & & \\
\hline
\end{tabular}

Table 3. Physical parameters for the condensations derived from the properties given in Table 2. Listed are: the excitation temperature $\left({ }^{12} T_{\text {ex }}\right)$ measured from the ${ }^{12} \mathrm{CO}(2-1)$ peak temperature; the ratio of ${ }^{12} \mathrm{CO}(2-1)$ and $(1-0)$ integrated intensities $\left({ }^{12} R\right)$; the ratio of ${ }^{13} \mathrm{CO}(2-1)$ and ${ }^{12} \mathrm{CO}(2-1)$ integrated intensities $\left({ }^{12,13} R\right)$; the ratio of $\operatorname{CS}(3-2)$ and $\mathrm{CS}(2-1)$ integrated intensities $\left({ }^{C S} R\right)$; ${ }^{13} \mathrm{CO}(2-1)$ optical depth $\left({ }^{13} \tau\right)$; ${ }^{13} \mathrm{CO}(2-1)$ column density $\left(N\left({ }^{13} \mathrm{CO}\right)\right) ; \mathrm{H}_{2}$ column density $\left(N\left(\mathrm{H}_{2}\right)\right)$; mass estimated under LTE conditions $\left(M_{\mathrm{LTE}}\right)$; Virial mass estimate $\left(M_{\text {virial }}\right)$ and the average $\mathrm{H}_{2}$ density $\left(n\left(\mathrm{H}_{2}\right)\right)$.

\begin{tabular}{lcccccccccccc}
\hline \hline Condensation & $\begin{array}{c}\text { Component } \\
\mathrm{km} \mathrm{s}^{-1}\end{array}$ & $\begin{array}{c}{ }^{12} T_{\text {ex }} \\
\mathrm{K}\end{array}$ & ${ }^{12} R$ & ${ }^{12,13} R$ & ${ }^{C S} R$ & ${ }^{13} \tau$ & $\begin{array}{c}N\left({ }^{13} \mathrm{CO}\right) \\
\mathrm{cm}^{-2}\end{array}$ & $\begin{array}{c}N\left(\mathrm{H}_{2}\right) \\
\mathrm{cm}^{-2}\end{array}$ & $\begin{array}{c}M_{\text {LTE }} \\
M_{\odot}\end{array}$ & $\begin{array}{c}M_{\text {virial }} \\
M_{\odot}\end{array}$ & $\begin{array}{c}n\left(\mathrm{H}_{2}\right) \\
\mathrm{cm}^{-3}\end{array}$ \\
\hline $\mathrm{G} 287.73-0.92$ & -27.8 & 21.7 & 1.34 & 5.43 & 1.00 & 0.20 & $7.0 \times 10^{15}$ & $2.8 \times 10^{21}$ & 11 & 84 & $4.1 \times 10^{3}$ \\
$\mathrm{G} 287.84-0.82$ & -14.2 & 39.5 & 1.64 & 3.88 & 0.89 & 0.40 & $21.5 \times 10^{15}$ & $8.6 \times 10^{21}$ & 34 & 197 & $12.5 \times 10^{3}$ \\
$\mathrm{G} 287.93-0.99$ & -19.8 & 29.0 & 2.15 & 2.64 & 0.58 & 0.37 & $14.6 \times 10^{15}$ & $5.7 \times 10^{21}$ & 45 & 145 & $16.6 \times 10^{3}$ \\
$\mathrm{G} 288.07-0.80$ & -21.6 & 20.7 & 1.26 & 4.07 & 0.96 & 0.16 & $7.0 \times 10^{15}$ & $2.8 \times 10^{21}$ & 11 & 134 & $4.1 \times 10^{3}$ \\
\hline
\end{tabular}

With the foreground extinction removed, the brightest sources will correspond to the most massive and youngest objects. Young sources display a near-IR excess, making them brighter in the $K_{\mathrm{s}}$-band than main-sequence stars whose emission is purely from a stellar photosphere. As stars tend to form in clusters, any over-densities within the stellar density distribution will potentially reveal the location of clusters. Figure 5 displays, as contours, the derived stellar density, and visual extinction maps for the southern Carina Nebula. Many overdensities are seen here and are candidate young clusters.

Coincident with a peak in the stellar density distribution is the known cluster Bo 11 and interestingly, two of the molecular condensations discussed in Sect. 3.5. The source G287.84-0.82 reveals, in 2MASS images, extended IR emission and a tightly packed group of sources, many of which are only visible in the $K_{\mathrm{s}}$-band image (this source will be discussed further in later sections).

Many other candidate clusters are identified from this analysis, but display no obvious clusters within the 2MASS images. Many are associated with peaks in the visual extinction maps, therefore making the identification of a cluster from the images difficult. While clusters are located across the region, it is those associated with the visual extinction peaks that are potentially the most interesting. This correspondence suggests the clusters may be embedded within the pillars. Additional data is required, however, to determine the exact nature of many of these candidate clusters.

\subsection{Identification of candidate YSOs}

Recently, Lumsden et al. (2002) derived criteria for identifying various galactic plane sources from fluxes obtained within the MSX bands. The main focus of their study was to separate massive young stellar objects (MYSOs) from sources with similar IR colours, in particular, compact $\mathrm{H}_{\text {II }}$ regions, young compact planetary nebulae, and very dusty evolved stars. From mid-IR colour-colour diagrams, they find that young objects satisfy $F_{21} / F_{8}>2$, with MYSOs having $F_{14} / F_{12}>1$, while compact $\mathrm{H}_{\text {II }}$ regions have $F_{14} / F_{12}<1$. These limits were determined from the location of a known sample of these objects. The sample of $\mathrm{H}_{\mathrm{II}}$ regions originated from sources identified within radio continuum surveys (Wood \& Churchwell 1989; Kurtz et al. 1994; Walsh et al. 1998). For the MYSOs, the list was compiled by Lumsden et al. (2002), and consisted of 


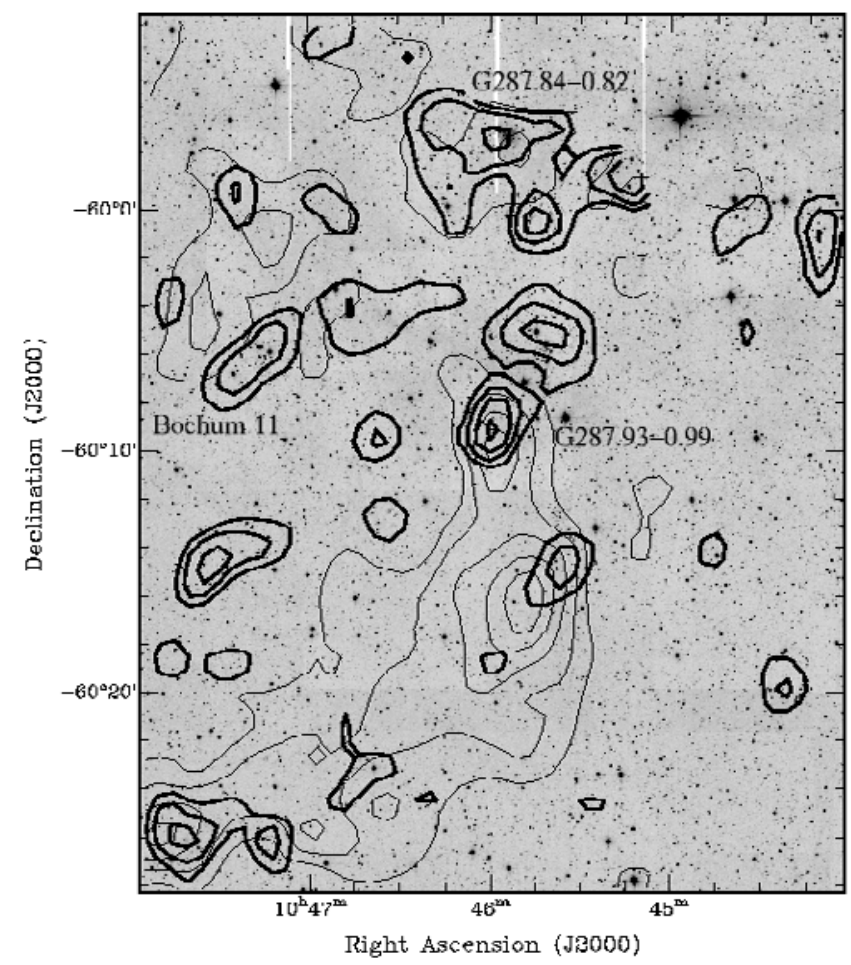

Fig. 5. $K_{\mathrm{s}}$-band image for the southern Carina Nebula with contours representing the stellar density distribution for stars with $K_{\text {corr }}<$ 12 mag (thick black: levels are 2.6, 3, 3.4, $3.8 \mathrm{stars} / \operatorname{arcmin}^{2}$ ) and the visual extinction map (thin black: levels are 7.5, 9.5, 11.5, 13.5 and $15.5 \mathrm{mag})$. The cluster Bo 11 is marked, along with the condensations G287.84-0.82 and G287.93-0.99.

objects with strong emission in the longer MSX bands, and with featureless mid-IR spectra, with the possible exception of silicate absorption. In this section, we use these criteria to investigate the nature of the point sources across the Carina Nebula in order to determine if, and where, any MYSOs and compact $\mathrm{H}$ II regions may exist.

Figure 6 shows the colour-colour diagram for all sources with reliable detections in the MSX and/or IRAS PSCs. Also included on this plot is the position of a black-body and the limits derived by Lumsden et al. (2002) for MYSOs and compact $\mathrm{H}_{\text {II }}$ regions.

In this colour-colour plane, sources with $F_{14} / F_{12} \leq 1$ and $F_{21} / F_{8} \leq 2$ correspond to evolved stars. The remaining sources that satisfy $F_{21} / F_{8}>2$, are candidate young objects, 13 of which fall within the compact $\mathrm{H}$ in region while 9 are potentially MYSOs. Visual inspection of the images reveal the sources displaying an $F_{21} / F_{8}>10$ are associated with diffuse emission and are not point sources (these are marked as filled squares in Fig. 6). Thus, their identification as MYSO objects is dubious. Two MYSO candidates remain and are located in the south of the nebula (labelled in Fig. 1 as G287.87-1.36 and G287.73-1.01). These appear isolated from any extended mid-IR, radio continuum emission, or the molecular condensations. A candidate compact $\mathrm{H}$ II region is, however, associated with one of the molecular condensations (G287.84-0.82).

Two sources identified within this analysis have previously been identified as YSOs and include a bright-rimmed globule at

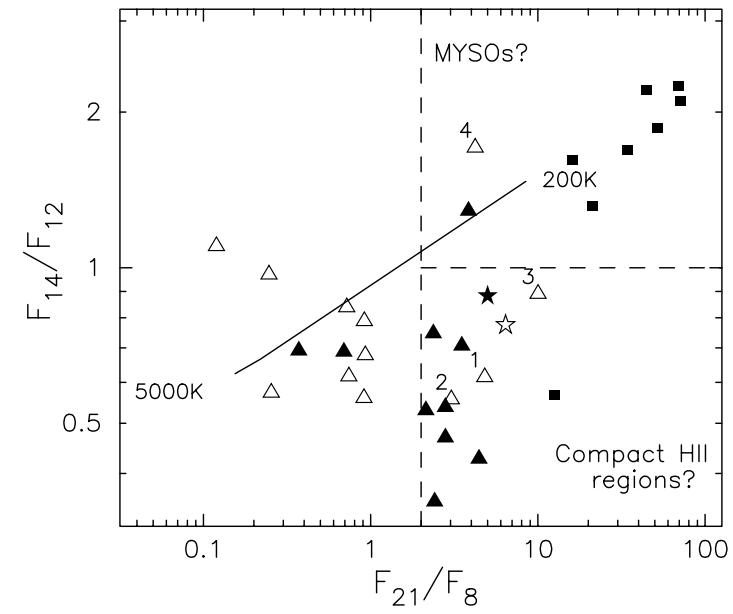

Fig. 6. Colour-colour plot for sources in the Carina Nebula. Included on this are the location of a black-body (solid line) and the limits derived by Lumsden et al. (2002) for MYSOs and compact H II regions (dashed lines). Plotted here are: point sources with $M S X$ data (filled triangles), point sources with MSX and IRAS detections (open triangles), MSX sources associated with diffuse emission (filled squares) and the source identified by Megeath et al. (1996) (open asterisk) and Rathborne et al. (2002) (filled asterisk). The numbers mark the sources discussed in Sect. 4.3.

the edge of the southern molecular cloud (marked with an open asterisk; G287.63-0.72; Megeath et al. 1996) and an embedded O9-star (filled asterisk; G287.47-0.54; Rathborne et al. 2002).

\subsection{Continuum SEDs}

To investigate the nature of the YSO candidates, spectral energy distributions (SEDs) were constructed. Of the 14 candidates, only 4 of these had reliable and coincident detections within both the MSX and IRAS PSCs. The first of these sources is located in the northern part of the nebula (G287.52-0.41), the second is the source associated with the molecular condensation (G287.84-0.82), the third is located at the tip of a giant pillar (G287.93-0.99), while the remaining source is located in the south, and is a MYSO candidate (G287.87-1.36). These are discussed in more detail in Sect. 4.5.

Figure 7 shows the continuum SEDs for these sources. Included on these plots are the fluxes obtained from the IRAS PSC, as well as those estimated from the MSX and 2MASS images. An additional $1.2 \mathrm{~mm}$ flux is included for the source G287.87-1.36. The solid line in each plot is a fit to the data using a grey-body of the form $B_{v}(T)\left(1-\exp \left(-\tau_{v}\right)\right) \Omega$, where $B_{v}(T)$ is the Planck function at a temperature $T, \tau_{v}$ is the dust optical depth, and $\Omega$ is the solid angle subtended by the dust shell. The opacity was assumed to have the form $\tau=\left(v / v_{0}\right)^{\beta}$ where $v_{0}$ is the frequency at which the emission becomes optically thick, with $\beta$ set to 2 (determined from tabulated values of Ossenkopf \& Henning 1994). A two-component model was fit to the data, because a single temperature component was insufficient to describe the observed emission. Parameters derived from these fits, including the temperatures and radii of the two dust components, mass, luminosity, and spectral type of the central star, are listed in Table 4. 


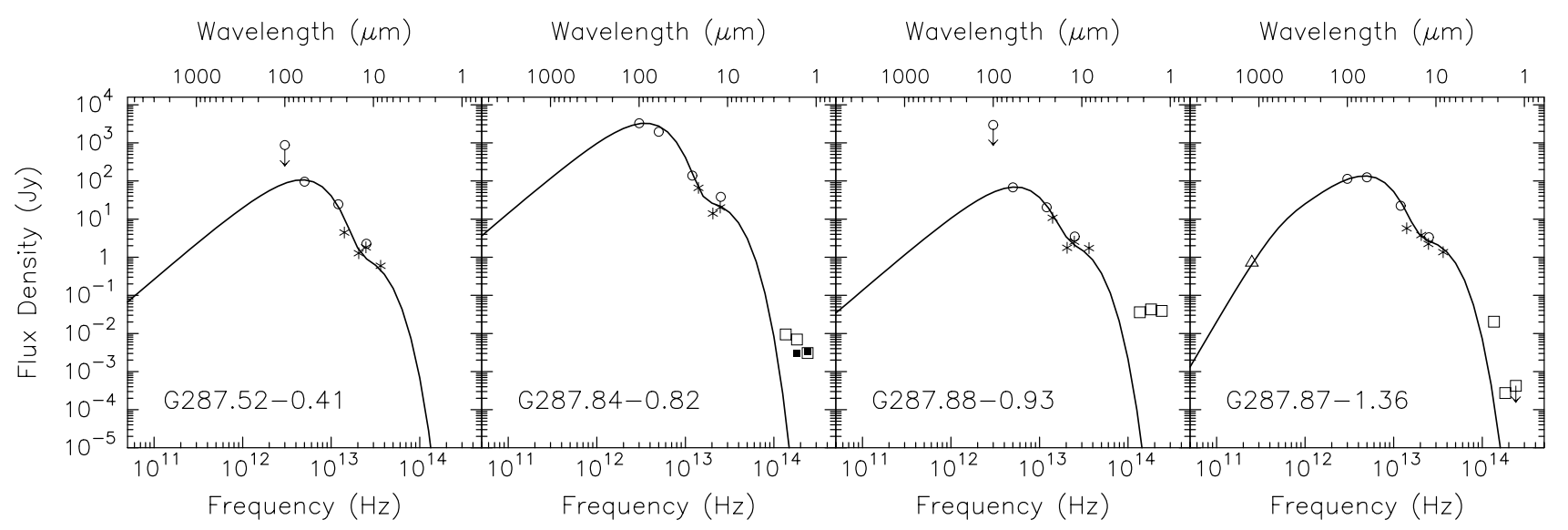

Fig. 7. Spectral energy distributions for the candidate YSOs; G287.52-0.41 (source 1, in Fig. 6), G287.84-0.82 (sources 2), G287.88-0.93 (source 3) and G287.87-1.36 (source 4). Open squares represent 2MASS fluxes, stars represent MSX fluxes, while circles mark the IRAS fluxes. The solid line in each plot is a fit to these points using a modified two component black-body function (see text for details). The $8 \mu \mathrm{m}$ flux is excluded from the fit for G287.84-0.82 as it is known to be dominated by emission from PAH molecules. The near-IR points were not included in the fits as these likely represent emission from an extincted hot component rather than dust emission.

Table 4. The derived parameters of the two-component grey-body fits to the SEDs for G287.52-0.41, G287.84-0.82 G287.88-0.93 and G287.87-1.36 as shown in Fig. 7. The parameters correspond to the temperatures of the outer $\left(T_{\text {outer }}\right)$ and inner $\left(T_{\text {inner }}\right)$ dust components, the inner $\left(R_{\text {inner }}\right)$ and outer $\left(R_{\text {outer }}\right)$ radii of the dust shell and estimates of the gas mass $\left(M_{\text {gas }}\right)$, Luminosity derived from these and the corresponding spectral types of the exciting star. The numbers in brackets next to the source names refer to the source label from Fig. 6.

\begin{tabular}{cccccccc}
\hline \hline Source & $T_{\text {outer }}$ & $\begin{array}{c}T_{\text {inner }} \\
\mathrm{K}\end{array}$ & $\begin{array}{c}R_{\text {inner }} \\
\mathrm{K}\end{array}$ & $\begin{array}{c}R_{\text {outer }} \\
\mathrm{AU}\end{array}$ & $\begin{array}{c}M_{\text {gas }}{ }^{a} \\
\mathrm{AU}\end{array}$ & $\begin{array}{c}\text { Luminosity } \\
M_{\odot}\end{array}$ & $\begin{array}{c}\text { Spectral } \\
10^{4} L_{\odot}\end{array}$ \\
\hline G287.52-0.41(1) & 80 & 320 & 10 & 850 & 16 & 5.1 & O8.5 \\
G287.84-0.82 (2) & 60 & 300 & 57 & 7200 & 1200 & 370 & cluster? \\
G287.88-0.93 (3) & 90 & 330 & 14 & 570 & 7.3 & 3.4 & B0 \\
G287.87-1.36 (4) & 80 & 360 & 14 & 960 & 12 & 3.3 & B0 \\
\hline
\end{tabular}

a The mass of G287.87-1.36 was determined from the observed flux at 1.2-mm. Estimates of the 1.2-mm flux for the other sources were obtained from the grey-body fit.

Observations at a frequency in which the emission is optically thin allows a determination of the mass of the material. For the source G287.87-1.36 additional data at 1.2-mm were obtained which, when included in fit, revealed a $v_{0}$ of $4.0 \times$ $10^{11} \mathrm{~Hz}(0.75 \mathrm{~mm})$. Thus, the emission at $1.2 \mathrm{~mm}$ is optically thin and was therefore used to estimate the mass. The expression $M_{\mathrm{gas}}=\frac{S_{v} D^{2}}{R_{\mathrm{dg}} B_{v}\left(T_{\mathrm{d}}\right)}$ was used, where $M_{\mathrm{gas}}$ is the total mass of an isothermal dust source, $S_{v}$ is the observed flux density at an optically thin frequency $v, \kappa_{v}$ is the mass absorption coefficient, $R_{\mathrm{dg}}$ is the dust-to-gas mass ratio and $B_{v}\left(T_{\mathrm{d}}\right)$ is the Planck function at the dust temperature (Chini et al. 1987). Using values of $R_{\mathrm{dg}} \sim 0.01$, a dust opacity of $0.1 \mathrm{~m}^{2} / \mathrm{kg}$ (determined from Ossenkopf \& Henning 1994), and assuming a distance of $2.2 \mathrm{kpc}$, the resulting mass was found to be $12 M_{\odot}$. The mass estimates represent a lower limit, as the dust opacity, and temperatures are uncertain, and are most likely lower than quoted here.

Luminosity estimates suggest the exciting stars for these sources are OB-stars. The high mass and luminosity derived for G287.84-0.82 suggest this object may harbour a cluster of stars.

\subsection{Near-IR three-colour images}

Figure 8 shows near-IR three-colour images constructed from the 2MASS data, for the 14 YSO candidates plus one source of interest (G287.86-0.82). This source was selected because of its extremely red near-IR colour, similar to the MYSO candidate G287.87-1.36. These images reveal any near-IR counterparts to the mid-IR sources, in particular, note the prominant cluster of reddened stars associated with G287.84-0.82.

Near-IR images of the sources G287.73-1.01 and G287.80-0.56, reveal bright stellar objects, the former being associated with a known O7-star (HD 93222; Walborn 1972). Although these objects lie in the regime for candidate MYSOs and compact $\mathrm{H}$ II regions respectively, they most likely represent older stars, as they are clearly seen in the near-IR and, for the object G287.73-1.01, also at visible wavelengths. As a result, these are not considered to be candidate YSOs.

Several sources identified as compact $\mathrm{H}_{\mathrm{II}}$ regions, are associated with diffuse emission in the near-IR images. These objects (G287.51-0.49, G287.22-0.53, G287.47-0.54, G287.63-0.72, G287.75-0.66, G287.76-0.87 and G287.68-1.09) all show extended $K_{\mathrm{s}}$-band emission, 

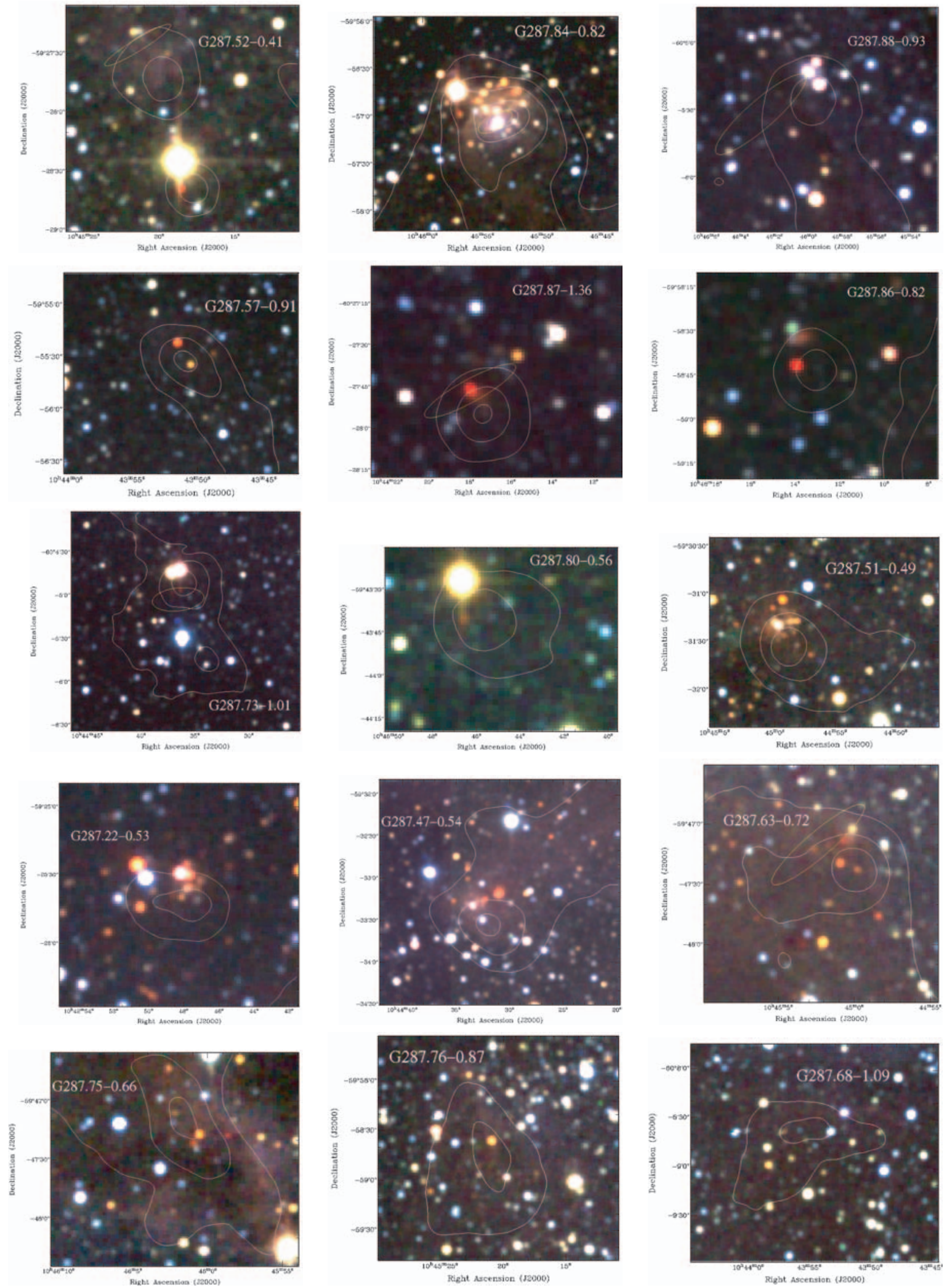

Fig. 8. A collection of near-IR three-colour images for candidate YSOs within the Carina Nebula ( $J$-band in blue, $H$-band in green and $K_{\mathrm{s}}$-band in red). The images are presented in the order in which they are discussed in Sect. 4.5. Included also are yellow ellipses marking the location of IRAS point sources and contours of $8 \mu \mathrm{m}$ emission. Levels are, G287.52-0.41: 0.5, 1; G287.84-0.82: 1, 2, 4, 8, 10; G287.88-0.93: 0.5, 1; G287.57-0.91: 0.5, 1, 1.5; G287.87-1.36: 0.5, 1, 2; G287.86-0.82: 0.5, 0.7; G287.73-1.01: 0.5, 1, 2; G287.80-0.56: 1, 2; G287.51-0.49: 1, 2, 3; G287.22-0.53: 0.8, 1.3; G287.47-0.54: 2, 4, 6; G287.63-0.72: 1, 2, 3; G287.75-0.66: 2, 3, 4; G287.76-0.87: 1, 2; G287.68-1.09: 1, 1.5. All are in units of $10^{-5} \mathrm{~W} \mathrm{~m}^{-2} \mathrm{sr}^{-1}$. 
with several reddened sources located at the peak of the $8 \mu \mathrm{m}$ emission. In addition, all seven of these objects have extended $843 \mathrm{MHz}$ radio continuum emission associated with them.

Four of these objects (G287.51-0.49, G287.22-0.53, G287.47-0.54, and G287.63-0.72) contain multiple sources and are possibly embedded clusters. These objects were all located external to the stellar density map of Sect. 4.1. The sources G287.47-0.54 and G287.63-0.72 have been discussed previously in Rathborne et al. (2002) and Megeath et al. (1996).

Near-IR images of the remaining three sources, G287.75-0.66, G287.76-0.87 and G287.68-1.09, show no obvious embedded stars, with the stellar colours most likely a result of interstellar extinction.

\subsection{Synopses of the most interesting YSO candidates}

\subsubsection{G287.52-0.41}

This source is located in the north of the Carina Nebula and has mid-IR colours indicative of a compact $\mathrm{H}$ II region (source 1 in Fig. 6). Consistent with this, is the detection of $843 \mathrm{MHz}$ radio continuum emission toward this object. Mass and luminosity estimates suggests the presence of an embedded O8.5-star. Furthermore, in the near-IR, a single reddened object is seen and is coincident with the MSX and IRAS point sources.

\subsubsection{The cluster within G287.84-0.82}

This is the only case within the current dataset, for which a clearly visible cluster of young stars has been found embedded within a molecular condensation (this cluster was first identified by Dutra \& Bica 2001). It is the brightest point source in the mid-IR and $843 \mathrm{MHz}$ radio continuum maps within the southern Carina Nebula. The near-IR three colour image for this cluster, reveals many embedded stars, with several detected only at the $K_{\mathrm{s}}$-band. These sources represent the most interesting and youngest within the cluster.

In the mid-IR colour-colour plane, this source (number 2), lies within the region for compact $\mathrm{H}_{\text {II }}$ regions. As expected, it shows strong, compact radio continuum emission. The mass and luminosity derived for this source, suggests it contains at least one massive star within a cluster of lower mass stars. This is consistent with previous studies which suggest a B1 star and reflection nebula exist here (ALS 1883; Herbst 1975). However, $6.7 \mathrm{GHz}$ methanol maser emission was not detected toward this object in a survey by MacLeod et al. (1998).

An averaged IRAS Low Resolution Spectrometer (LRS) spectra was also obtained for this object (Fig. 9). Within the sizeable LRS aperture $\left(5^{\prime}\right.$ at $8-13 \mu \mathrm{m}$ and $7.5^{\prime}$ at $\left.11-23 \mu \mathrm{m}\right)$ strong PAH emission is seen at 7.7 and $11.3 \mu \mathrm{m}$, confirming our previous identification of PAHs on the basis of the MSX and ${ }^{12} \mathrm{CO}$ morphology. Flux densities estimated from the MSX images and PSC, and IRAS PSC, are also included on this plot. The obvious discrepancies between flux densities derived from the LRS, the MSX image, and those from the MSX PSC, reflect the complexity of the region, with its widespread mid-IR

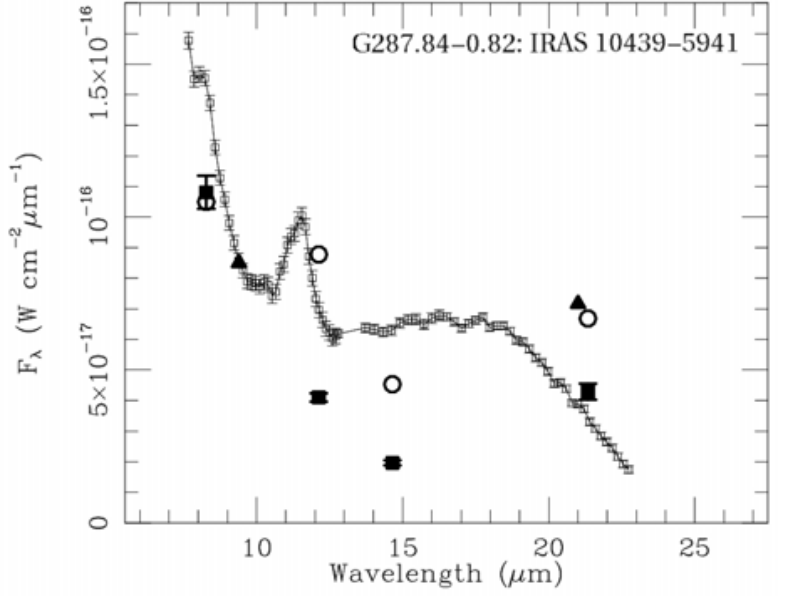

Fig. 9. The IRAS LRS spectrum of G287.84-0.82 (open squares) with error bars, and comparisons with photometry from the IRAS PSC (filled triangles) and MSX PSC (filled squares) and from the MSX images (open circles).

emission, and the difficulties in choosing an appropriate background level to subtract from the dominant mid-IR peak.

\subsubsection{G287.88-0.93 and G287.57-0.91}

The mid-IR morphology of these two sources show filamentary features with point sources at their tips. They were included within the colour-colour plot of Fig. 6 and fall within the regime for compact $\mathrm{H}$ II regions. However, no strong $843 \mathrm{MHz}$ radio continuum emission is associated with them. The source G287.88-0.93 does, however, contain an IRAS point source, with its SED corresponding to an embedded B0 star. A star of this spectral type would produce a compact $\mathrm{H}_{\text {II }}$ region, albeit weak, and possibly below the detection limits of the MOST considering the distance to the nebula.

The near-IR image for G287.88-0.93 shows a group of three stars falling within the peak of the $8 \mu \mathrm{m}$ emission. NearIR images of G287.57-0.91, reveal a bright $K_{\mathrm{s}}$-band source located at the tip of the filamentary $8 \mu \mathrm{m}$ feature.

\subsubsection{G287.87-1.36}

This source is the most likely to correspond to a bona-fide MYSO, as it lies within this regime in the mid-IR colourcolour diagram, and has a corresponding IRAS point source. The luminosity derived from its SED suggests an embedded B0-star. An extremely red object exists in the near-IR three colour image, and is coincident with an unresolved mid-IR and 1.2-mm continuum source. The IRAS LRS for this source (Fig. 10), clearly shows a deep silicate absorption profile at $10 \mu \mathrm{m}$ indicative of high extinction.

In addition, Bronfman et al. (1996) detected CS (2-1) line emission toward this object in their survey of IRAS sources having colours of compact $\mathrm{H}_{\mathrm{II}}$ regions. It appears in their Table 1 as IRAS 10423-6011, with $V_{\mathrm{lsr}}=13.7 \mathrm{~km} \mathrm{~s}^{-1}, T_{\mathrm{mb}}=$ $0.84 \mathrm{~K}$ and $\Delta V=2.7 \mathrm{~km} \mathrm{~s}^{-1}$. Because the central velocity is blue-shifted by more than $15 \mathrm{~km} \mathrm{~s}^{-1}$ with respect to the other 


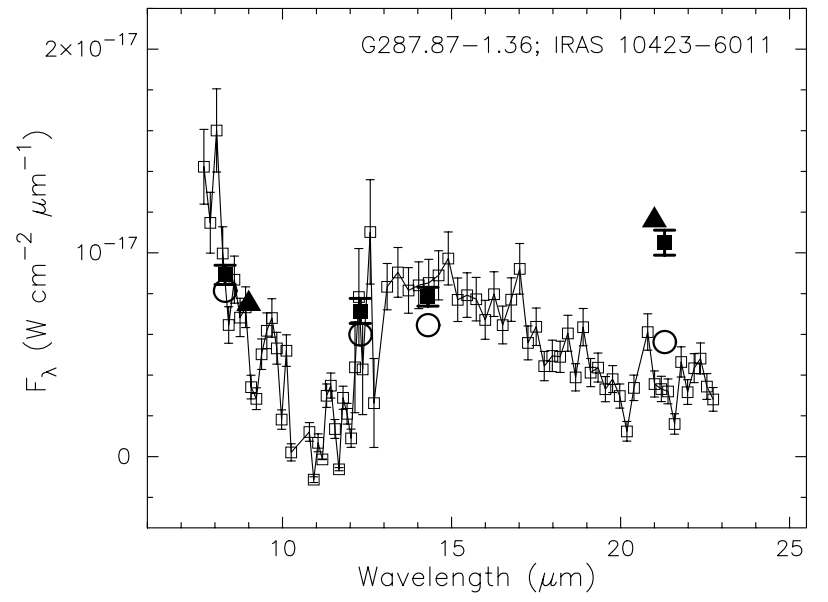

Fig. 10. The IRAS LRS spectrum of G287.87-1.36 (open squares) with error bars, and comparisons with photometry from the IRAS (filled triangles) and MSX (filled squares) PSCs and from the MSX images (open circles).

molecular condensations here, it is not clear if this object is actually associated with the nebula.

\subsubsection{G287.86-0.82}

Located to the east of the cluster G287.84-0.82 is the object G287.86-0.82. This source shows weak $8 \mu \mathrm{m}$ emission, with a strong $K_{\mathrm{s}}$-band source. Located nearby, is a second object whose emission peaks in the $H$-band with extended $K_{\mathrm{s}}$-band emission (green in the near-IR three colour image). Such emission either arises from a reflection nebula or shocked gas. Detailed spectra are needed to determine the exact nature of this object.

\subsection{Ongoing star formation within the pillars}

The sources identified here significantly increase the number of young objects found within the Carina Nebula. Previous studies located 6 embedded OB-stars in the northern molecular cloud complex associated with $\operatorname{Tr} 14$ (Brooks et al. 2001; Rathborne et al. 2002), while a single B0 star is seen at the edge of the southern molecular cloud (Megeath et al. 1996).

To investigate the age distribution of the star formation within this GMC, the recent rate of massive star formation $(\psi)$ has been estimated. Studies of the stellar population within the clusters $\operatorname{Tr} 14$ and $\operatorname{Tr} 16$ find that the massive stars formed within the past 1-3 Myr (DeGioia-Eastwood et al. 2001; Tapia et al. 2003). These clusters have a population of $\sim 33$ O-type stars, which implies a $\psi$ of $\sim 1-3 \times 10^{-5} \mathrm{O}$-stars $\mathrm{yr}^{-1}$.

Mass and luminosity estimates exist for ten young objects within the Carina Nebula (the seven previously identified and the three additional sources identified here). Age estimates were calculations from their Kelvin-Helmholtz time, assuming they consist of a single star. Ages were found to range between $1-5 \times 10^{5} \mathrm{yrs}$, which for these 10 sources, suggests $\psi$ to be $\sim 3 \times 10^{-5} \mathrm{O}$-stars $\mathrm{yr}^{-1}$. This value is consistent with the rate determined from the known optical O-stars and is expected if the rate of star formation has been constant over the age of the nebula. While this is possible, these two epochs may instead represent two isolated bursts of star formation. Distinguishing between these two mechanisms is difficult with the limited data presented here.

The rate of star formation for the Carina Nebula is comparable with estimates for two other well studied regions of massive star formation. For instance, NGC 3606 contains 50 O-type stars which have formed over the last 2-3 Myrs (see Eisenhauer et al. 1998 and references therein). This suggests a $\psi$ of $\sim 1-3 \times 10^{-5} \mathrm{O}$-stars $\mathrm{yr}^{-1}$. While for 30 Dor, at a similar age, and containing 100 O-type stars Walborn \& Blades (1997), the rate is estimated to be $\sim 3-5 \times 10^{-5} \mathrm{O}$-stars $\mathrm{yr}^{-1}$.

\section{Conclusions}

We have undertaken a multi-wavelength study incorporating data from 2MASS, MSX, IRAS, MOST, and the SEST, to investigate the nature of, and search for star formation within, the giant pillars of the Carina Nebula. Our main results and conclusions are summarised below.

\subsection{Molecular clouds, PDRs and ionization fronts}

Emission from the $8 \mu \mathrm{m} M S X$ band outlines the known molecular clouds and giant pillars within the Carina Nebula, and pinpoints regions where the UV radiation is penetrating the molecular material and forming PDRs. Visual extinction maps match extremely well the $8 \mu \mathrm{m}$ emission tracing the dense gas associated with the pillars.

Interestingly, the $21 \mu \mathrm{m}$ and $843 \mathrm{MHz}$ radio continuum emission match extremely well and are located along the edges of the PDRs closest to the most influential clusters. Emission within these bands reveals heated $\sim 40 \mathrm{~K}$ dust and ionization fronts. The geometry of the largest pillar is consistent with interactions from the nearby massive stars carving the molecular material around a dense core.

Bright condensations located at the tips of the giant pillars, were found to be externally heated, with PDRs located along the edges in the direction of the massive clusters. The properties of the molecular material suggest they have the potential to be massive star forming cores.

\subsection{Evidence for recent star formation activity}

To search for evidence of star formation activity a stellar number density map was used. This was derived from the dereddened $K_{\mathrm{s}}$-band magnitudes for sources in the 2MASS PSC. Many candidate young clusters were identified within this map, several of which appear to be related to, and possibly embedded within, the giant pillars. Interestingly, the brightest IR and radio continuum source in the region contained the only case of a clearly visible cluster within the 2MASS images.

A candidate MYSO and several compact $\mathrm{H}$ II regions were identified across the nebula using mid-IR colour criteria. SEDs were constructed to study these objects further, and reveal the 
Table 5. Coordinates for all sources discussed (taken from MSX data). Comment descriptions include: massive young stellar object candidate (MYSO); compact $\mathrm{H}_{\text {II }}$ regions ( $\mathrm{H}_{\text {II }}$ ); bright stellar object (S); tip of a filamentary feature (T); molecular condensation (MC); cluster (C); diffuse $K_{\mathrm{s}}$-band emission (DE); red object in 2MASS images (R) and any additional nomenclature. The divisions in the table separate the MYSO, the compact $\mathrm{H}_{\text {II }}$ regions, the sources that most likely correspond to evolved stars, the source displaying an extremely red colour in 2MASS images, and the molecular condensations. Fluxes for these sources are given in Rathborne (2003).

\begin{tabular}{|c|c|c|c|}
\hline \multirow[t]{2}{*}{ Source } & \multicolumn{2}{|c|}{ Coordinates } & \multirow[t]{2}{*}{ Comments } \\
\hline & $\alpha_{J 2000}$ & $\delta_{J 2000}$ & \\
\hline \multirow[t]{2}{*}{ G287.87-1.36 } & 104417.9 & -602746 & MYSO; B0-star \\
\hline & & & IRAS 10423-6011 \\
\hline \multirow[t]{2}{*}{ G287.52-0.41 } & 104520.9 & -592728 & H II; O8.5-star \\
\hline & & & IRAS 10434-5911 \\
\hline \multirow[t]{3}{*}{ G287.84-0.82 } & 104553.6 & -595710 & H II; MC; C; \\
\hline & & & OB-cluster; \\
\hline & & & IRAS 10439-5941 \\
\hline \multirow[t]{2}{*}{ G287.88-0.93 } & 104600.9 & -600512 & Н II; T; B0-star \\
\hline & & & IRAS 10441-5949 \\
\hline G287.57-0.91 & 104351.1 & -595523 & $\mathrm{H}_{\text {II }} ; \mathrm{T}$ \\
\hline G287.51-0.49 & 104459.0 & -593124 & $\mathrm{H}_{\text {II }} ; \mathrm{DE}$ \\
\hline G287.22-0.53 & 104249.2 & -592527 & $\mathrm{H}_{\text {II; }} \mathrm{DE}$ \\
\hline $\mathrm{G} 287.47-0.54^{a}$ & 104432.8 & $-59: 33: 20$ & $\mathrm{H}_{\text {II }} ; \mathrm{DE}$ \\
\hline \multirow[t]{2}{*}{$\mathrm{G} 287.63-0.72^{b}$} & 104501.1 & -594706 & $\mathrm{H}_{\mathrm{II}} ; \mathrm{DE}$ \\
\hline & & & IRAS 10430-5931 \\
\hline G287.75-0.66 & 104601.2 & -594658 & $\mathrm{H}_{\text {II; }}$ DE \\
\hline G287.76-0.87 & 104522.3 & -595823 & $\mathrm{H}_{\text {II }}$ \\
\hline G287.68-1.09 & 104357.2 & -600825 & $\mathrm{H}_{\text {II }}$ \\
\hline G287.73-1.01 & 104434.6 & -600536 & S; HD 93222 \\
\hline G287.80-0.56 & 104646.8 & -594325 & $\mathrm{~S}$ \\
\hline G287.86-0.82 & 104613.9 & -595841 & $\mathrm{R}$ \\
\hline G287.73-0.92 & 104445.4 & -595916 & $\mathrm{MC}$ \\
\hline G287.93-0.99 & 104556.1 & -600850 & $\mathrm{MC} ; \mathrm{C} ?$ \\
\hline G288.07-0.80 & 104735.4 & -600251 & $\mathrm{MC}$ \\
\hline
\end{tabular}

${ }^{a}$ Source N4 from Rathborne et al. (2002).

${ }^{b}$ Identified by Megeath et al. (1996).

exciting stars in several cases correspond to OB-stars. Table 5 lists a summary of all the candidates.

\subsection{Triggered star formation in the pillars?}

The results presented here clearly show that the large molecular clouds within the Carina Nebula are being strongly affected by the intense stellar winds and harsh radiation fields from the nearby massive star clusters. For instance, giant pillars are forming as a result of these interactions, as dense cores shield the surrounding material. What still remains unclear however, is the effect these interactions have on the ongoing star formation within this GMC. Young clusters and candidate MYSOs are found both within, and external to, the giant pillars. In addition, while star formating sites are found scattered across the region, there are several locations where the intense interactions from the known clusters are potentially triggering the activity. It is also likely that the rate of star formation has been constant within the nebula since the birth of the massive star clusters.

Acknowledgements. JMR and MGB acknowledge the support of the Australian Research Council and the University of New South Wales. MC thanks NASA for its support of his participation in this work under LTSA grant NAG5-7936 with the University of California, Berkeley. This publication makes use of data products from 2MASS (a joint project of the UMASS and the IPAC/CIT, funded by NASA and the NSF) and MSX (processing of this data was funded by the Ballistic Missile Defense Organization with additional support from NASA's Office of Space Science).

\section{References}

Bronfman, L., Nyman, L.-A., \& May, J. 1996, A\&AS, 115, 81

Brooks, K. J., Storey, J. W. V., \& Whiteoak, J. B. 2001, MNRAS, 327 , 46

Brooks, K. J., Whiteoak, J. B., \& Storey, J. W. V. 1998, Proc. Astron. Soc. Aust., 15, 202

Buisson, G., Desbats, L., Duvert, G., et al. 1999, GILDAS, see http://iram.fr/GS/gildas.html

Chini, R., Kruegel, E., \& Wargau, W. 1987, A\&A, 181, 378

Cohen, M., \& Green, A. 2001, MNRAS, 325, 531

Cox, P., \& Bronfman, L. 1995, A\&A, 299, 583

de Graauw, T., Lidholm, S., Fitton, B., et al. 1981, A\&A, 102, 257

DeGioia-Eastwood, K., Throop, H., Walker, G., \& Cudworth, K. M. 2001, ApJ, 549, 578

Dutra, C. M., \& Bica, E. 2001, A\&A, 376, 434

Egan, M. P., Shipman, R. F., Price, S. D., et al. 1998, ApJ, 494, L199

Eisenhauer, F., Quirrenbach, A., Zinnecker, H., \& Genzel, R. 1998, ApJ, 498, 278

Feinstein, A. 1995, Rev. Mex. Astron. Astrofis. (Ser. Conf.), 2, 57

Frerking, M. A., Langer, W. D., \& Wilson, R. W. 1982, ApJ, 262, 590

Gardner, F. F., \& Morimoto, M. 1968, ApJ, 21, 881

Harvey, P. M., Hoffmann, W. F., \& Campbell, M. F. 1979, ApJ, 227 , 114

Herbst, W. 1975, j, 80, 212

Hollenbach, D., \& Tielens, A. G. G. M. 1999, Rev. Mod. Phys., 71, 173

Jiang, Z., Yao, Y., Yang, J., et al. 2002, ApJ, 577, 245

Kurtz, S., Churchwell, E., \& Wood, D. O. S. 1994, ApJS, 91, 659

Lada, C. J., Lada, E. A., Clemens, D. A., \& Bally, J. 1994, ApJ, 429, 694

Lumsden, S. L., Hoare, M. G., Oudmaijer, R. D., \& Richards, D. 2002, MNRAS, 336, 621

MacLeod, G. C., van der Walt, D. J., North, A., et al. 1998, AJ, 116, 2936

McCaughrean, M. J., \& Andersen, M. 2002, A\&A, 389, 513

Megeath, S. T., Cox, P., Bronfman, L., \& Roelfsema, P. R. 1996, A\&A, 305, 296

Mill, J. D., O’Neil, R. R., Price, S., et al. 1994, J. Spacecraft Rockets, 31, 900 
Mills, B. Y. 1981, Proc. Astron. Soc. Aust., 4, 156

Ossenkopf, V., \& Henning, T. 1994, A\&A, 291, 943

Price, S. D., Egan, M. P., Carey, S. J., Mizuno, D., \& Kuchar, T. 2001, AJ, 121, 2819

Price, S. D., Tedesco, E. F., Cohen, M., et al. 1996, Bull. Am. Astron. Soc., 28, 1341

Rathborne, J. M. 2003, Ph.D. Thesis, University of New South Wales, Australia

Rathborne, J. M., Burton, M. G., Brooks, K. J., et al. 2002, MNRAS, 331,85

Retallack, D. S. 1983, MNRAS, 204, 669

Robertson, J. G. 1991, Austr. J. Phys., 44, 729

Smith, N., Egan, M. P., Carey, S., et al. 2000, ApJ, 532, L145

Stanke, T., Smith, M. D., Gredel, R., \& Szokoly, G. 2002, A\&A, 393, 251
Tapia, M., Roth, M., Vázquez, R. A., \& Feinstein, A. 2003, MNRAS, 339,44

Tovmassian, H. M. 1995, Rev. Mex. Astron. Astrofis. (Ser. Conf.), 2, 83

Verstraete, L., Pech, C., Moutou, C., et al. 2001, A\&A, 372, 981

Walborn, N. R. 1972, aj, 77, 312

Walborn, N. R., \& Blades, J. C. 1997, ApJS, 112, 457

Walsh, A. J., Burton, M. G., Hyland, A. R., \& Robinson, G. 1998, MNRAS, 301, 640

Whiteoak, J. B., \& Otrupcek, R. E. 1984, Proc. Astron. Soc. Aust., 5, 552

Whiteoak, J. B. Z. 1994, ApJ, 429, 225

Wood, D. O. S., \& Churchwell, E. 1989, ApJ, 340, 265

Zhang, X., Lee, Y., Bolatto, A., \& Stark, A. A. 2001, ApJ, 553, 274 